\title{
8 Research access to in-copyright texts in the humanities
}

\author{
Peter Organisciak and J. Stephen Downie
}

\section{Introduction}

In 2004, John Unsworth noted that the primary constraint to humanities in the digital age is the current copyright landscape, limiting which primary sources can be accessed, shared and studied. He argued that approaches "which deal with texts in the aggregate... provide a way around the copyright constraint" (2004). Since then, the emergence of massive digital collections has presented extensive opportunities for digital humanists and invigorated large-scale study of the published archive beyond the traditional canon. As predicted, though, the indexing, access and use of digital collections has remained limited by intellectual property challenges and legal frameworks, while beginning to address text mining, have been cautious and unassertive in adapting to such modes of inquiry.

Quantitative text analysis presents efficient and sometimes wholly new approaches to the study of culture and history, as seen in digital humanities work in stylometrics (e.g., Holmes 1985), culturomics (Michel et al. 2011), distant reading (Moretti 2013) and cultural analytics (Manovich 2009). However, access remains a problem, as the scale that makes digital methods promising is also untenable in current intellectual property systems. Due to information access issues, it is significantly more difficult to perform digital humanities work on the past century of text (Ross and Sayers 2014). This leads to a situation where some eras of study are more privileged in the digital humanities than others.

This chapter pursues the question, what information access approaches can enable research use of privileged or sensitive corpora? Increasingly, the solutions being pursued fall under the approach of non-consumptive access or non-expressive use (Jockers, Sag, and Schultz 2012), which describe uses that do not require a full text to be closely read or distributed. As Unsworth speculated, "if we don't have to republish work in order to do digital humanities, perhaps we can get at a greater portion of that record" (2004). This chapter will focus on text abstraction techniques, which represent a text through text patterns and statistics. The approach is viewed through the 
efforts of the HathiTrust Research Center (HTRC) in providing mediating scholarly use of 17 million digitised library volumes.

Non-consumptive approaches to information access present some challenges. Texts can lose important context and not all scholarly uses are served by the particular choices made in preparing the intermediate resource. However, they also present a path towards the study of modern texts, where the alternatives are either highly restricted or none at all. As the text analysis subdomains of the digital humanities mature, striking a balance between access and expressiveness of research texts will be vital and the current landscape of work will inform those choices.

The remainder of this chapter considers the value that growing digital libraries hold for scholarship, and how that value is challenged by legal hurdles. First, we consider the broader landscape of digital library growth, large-scale study of primary sources and the role of copyright in shaping that scholarship. Then we turn to the instructive example of the HTRC, to consider how one centre is addressing these challenges. The HTRC supports research on the 17 million digitised bibliographic volumes of HathiTrust Digital Library, of which 10.5 million volumes cannot be distributed because of their copyright status. In aggregate, it is a massive resource for digital humanities research into historical, cultural and linguistic trends. The HTRC was founded to encourage that potential through non-consumptive uses of the corpus, and serves as a case study in how scholarship over in-copyright corpora may grow.

\section{Background}

Computational analysis of text has been a central method in the emergence of what came to be known as humanities computing and eventually, the digital humanities. If the humanities are primarily a discipline of appreciation and understanding of cultural production, computing has offered new lenses and reconfigurations through which we may view our primary sources.

Early in the history of humanities computing, text digitisation was done in service of analysis. Some of the earliest known digitised texts were the Revised Standard Version of the Bible and the works of Thomas Aquinas, for projects led by Rev. John W. Ellison and Fr. Busa (Burton 1981, Hockey 2004). Both cases were in service of concordances, time-intensive reconfigurations of texts that the leaders of both projects believed could be better done by computers. "By using means that no age before ours could afford", a review of Busa's Index Thomisticus noted, "we are able now to arrive at that complete knowledge of Aquinas" (Sprokel 1978).

Computational analysis of texts and other primary sources has branched into various subdomains. With stylometrics (e.g., Holmes 1985, Burrows 1987), scholars sought to quantify authorial style, both towards better 
understanding the author's stylistic markers as well as to better appreciate their writing. With distant reading (Moretti 2013), scholars sought to position the value of broader, quantified analysis of corpora, as a complement to traditions of close reading. Such an approach has grown to massive scales in the context of cultural analytics for humanistic inquiry (Manovich 2009), and culturomics for inferential, corpus-driven analysis (Michel et al. 2011). These sub- and related domains have longer traditions in the humanities and social sciences (Underwood 2017), but in many cases have been invigorated by computational affordances, which excel in quantifying with consistency and scaling.

Accompanying the maturation of computation text analysis has been an emergence of large primary-text corpora, both born-digital and digitised. Contemporary materials are readily available from the digital age, with web archiving offering a new tool for historians (Milligan 2016, Milligan 2019) and user-generated content and social media providing material for cultural theory, ethnology and other social sciences (Manovich 2009, Lazer et al. 2009). Digital collections are large, and will continue to grow. As of late 2020, the Internet Archive, which preserves media as well as web pages, stores 70 petabytes of data and 475 billion web pages (Jessen 2020). Common Crawl, a repository of web data designed for programmatic access, contains 280 terabytes with 2.7 billion web pages in 2021 (Common Crawl 2021). The Library of Congress's Twitter Archive collected 12 years of messages before turning to more selective archive in 2018 (Osterberg 2017).

Given the effort involved, corpora of digitised materials have been slower to emerge, though with some notable standouts. LexisNexis has been providing electronic access to news and legal material since the 1970s (LexisNexis 2003) and JSTOR has digitised 12 million academic journals since 1994, for example. Through federated or consortial efforts, however, recent years have seen more collections of such scale become accessible for scholarship. In 2008, Europeana was launched as a cultural heritage digital library, starting with 4.5 million digital objects from over 1,000 European museums, archives and other institutions (Purday 2009). Similar efforts have been undertaken in the United States with IMLS Digital Collections and Content (Palmer, Zavalina, and Mustafoff 2007) and the Digital Public Library of America (Darnton 2013), both collecting cultural heritage materials from multiple institutions.

For years, the most notable bibliographic collection was Project Gutenberg. Founded at the University of Illinois in 1971, the project's volunteers have hand-transcribed 60,000 public domain works. The real inflection point, however, was with the Google Books project in the early 2000s. Unlike previous projects, which had to be selective for the costly and time-intensive process of digitisation, Google aimed to scan as many books as possible, partnering with predominantly academic, US-based libraries 
to scan millions of books, sharing the scans with the institutions. They also showed willingness to partner with researchers, notably releasing the Google NGrams Viewer and Dataset while supporting founding research on culturomics (Michel et al. 2011).

In response to the Google Books project, other consortial digitisation projects were founded. The Open Content Alliance was conceived by the Internet Archive, with support from Yahoo and Microsoft (Suber 2005). The Internet Archive has continued with its own digitisation initiatives, which has grown to over 2 million books (Freeland 2021). Finally, the HathiTrust was founded as a non-profit consortium, to separately collect works from different institutions' collections - including a significant portion that had been digitised by Google.

In this chapter, we focus on access to the HathiTrust collection, so we first turn to characterising the collection.

\section{About the HathiTrust}

The HathiTrust is a consortium collecting scanned works of institutions around the world in the massive HathiTrust Digital Library. The library is bibliographic in nature, primarily consisting of scanned books, as well as pamphlets, serials and other text materials; here, we adopt the HathiTrust's general language referring to those scanned text materials as volumes. Related works, such as identified duplicates and parts of a multi-volume set, are represented together in catalogue records.

As of 2021, the HathiTrust contains 17.4 million scanned volumes, representing 8.9 million unique catalogue records and contributed from over a hundred institutions worldwide (HathiTrust 2021a). Its roots lie in the Google Books project, a large digitisation project which sought access to academic library collections for scanning in exchange for providing the libraries the scans of those works. As individual institutions' digital collections grew from this effort, the HathiTrust was founded to allow a central, non-profit place for preservation and access of digitised works, with the Google-digitised materials being a large component of that collection. Today, those materials are still a significant portion of the HathiTrust Digital Library and the HathiTrust still maintains an ongoing relationship with Google's effort.

While the US institutions and the English language are most notably represented in the collection, there is still broad coverage beyond them. A number of institutions from Australasia and Europe contribute to the HathiTrust, and about $50 \%$ of the collection is in languages other than English ("HathiTrust Languages" 2021c). Of these, 800,000 catalogue records are in German (8.6\%), 650,000 are in French (7\%) and 608,000 are in Spanish $(6.5 \%)$, followed by Russian (3.3\%), Chinese (3\%), Japanese (2.6\%), Italian $(2.6 \%)$, Arabic $(1.5 \%)$ and Portuguese $(1.5 \%)$. Overall, there are 465 unique languages represented, 41 of which contain more than 10,000 volumes. 
The HathiTrust Digital Library collection also has broad topical and temporal coverage. Among the 5.0 million catalogue records that have an assigned Library of Congress Classification number, all 21 classes are represented, with a median of 136,000 records per class. Temporally, the collection spans multiple centuries, with $1.75 \%$ of volumes from the 18 th century or earlier, $14.25 \%$ from the 19 th century, $74.36 \%$ from the 20 th century and $9.65 \%$ from the 21 st century.

Given that the collection spans eras, subjects and languages while still maintaining large sample sizes in various slices of the collection, the HathiTrust presents tremendous value for the digital humanities. In addition to its value for finding individual primary materials for scholarship, the pairing of scale with digital access can support emerging forms of large-scale, aggregate-level corpus scholarship. This type of work is embodied in overlapping and variant subdomains including culturomics (Michel et al. 2011), distant reading (Moretti 2013) and cultural analytics (Manovich 2009).

\section{Challenges to computational text analysis at scale}

The challenges to scholarly use of corpora such as Google Books, HathiTrust and the Internet Archive are curatorial and organisational. In some instances, making sense of what is in the collection is important for contextualising aggregate-level inferences. For example, given that the contributing institutions to the HathiTrust are predominantly academic, there is a higher representation of scientific works. It's been noted, for example, that the capital-F Figure occurs more than figure in Google Books (Pechenick, Danforth, and Dodds 2015) and in turn, the HathiTrust. Given that the digital library materials are associated with professionally catalogued metadata, it is possible to account for content biases by focusing on specific subject classes or subsampling from a balanced subset of them, but understanding the biases of the collection is the first step. A further curatorial challenge is simply sifting through the scale to find quality materials. For example, there is uneven but pervasive duplication in the collection, which can be detrimental to certain types of text analysis (Schofield, Thompson, and Mimno 2017) and is not a trivial problem to address (Organisciak et al. 2019).

Of the challenges facing scholars in working with digitised collections, however, the greatest is legal. In the largest collections, a great many of the works are still in copyright, in the United States and around the world, and cannot easily be distributed to scholars or - in some jurisdictions - even studied by them. While challenges of curation, access and scale are tractable with enough effort and resources, the legal problem is more pernicious. It presents a barrier which, short of copyright reform, needs to be worked around rather than overcome. 


\section{Case study}

The HTRC was founded to address the varying challenges of scale, access, curation and copyright inherent to using the HathiTrust collection. This section focuses on how the HTRC uses non-consumptive access to navigate the primary issue of enabling research over sensitive documents. We consider the legal challenges to brokering access to the collection, then examine two approaches that the centre takes in working within those restraints: the Extracted Features (EF) Dataset and the exploratory data analysis tool HathiTrust+Bookworm (HT+BW). Finally, we consider how their model may apply to other digital libraries.

\section{HathiTrust Research Center}

While the HathiTrust Digital Library serves to meet traditional tenets of libraries, improving preservation and access to primary materials, the value of the collection as data was acknowledged with the founding of the HTRC, as a partnership between the University of Illinois and Indiana University. The Research Center was founded to support scholarship over the collection, particularly the type of large-scale study that the collection is uniquely positioned to support. As noted in their Non-Consumptive Use Policy, the HTRC "leverages the scope and scale of the repository to develop avenues for nonconsumptive research of the HathiTrust Digital Library" (HathiTrust 2017).

\section{Copyright considerations and non-expressive access}

The HTRC's approach to in-copyright materials is motivated by two key considerations:

\section{A substantial portion of the HathiTrust \\ Digital Library is contemporary}

According to the HathiTrust, nearly two-thirds of the digital library collection $(63.6 \%)$ is composed of materials from 1950 onward (HathiTrust 2021b). This includes $9.8 \%$ of materials created in the $1960 \mathrm{~s}, 12.1 \%$ from the $1970 \mathrm{~s}, 13.8 \%$ from the $1980 \mathrm{~s}, 13.1 \%$ from the $1990 \mathrm{~s}$ and $9.6 \%$ from the new millennium. There are millions of works in the HTDL from earlier in that timeframe. Yet, the heavy representation in the latter 20th century suggests that use of the collection should support contemporary, as well as classical works. A research centre that focuses on older, out-of-copyright works is neglecting the majority of the collection.

\section{The HathiTrust serves an international audience of patrons, scholars and researchers.}

The HathiTrust's constituents and contributors are from around the world, a fact that may be overlooked when noting its home at the University of 
Michigan. Further, the English materials comprise a bare majority, at about half of the full collection, with over 400 languages represented. As such, the HathiTrust requires international considerations, and in turn must navigate many different legal systems.

From a copyright standpoint, accommodating an international audience complicates any scholarly use mission. The US copyright system is restrictive to a degree, with longer copyright terms than many countries. Yet, it has two characteristics that simplify working with an era-spanning collection like the HTDL, at least in the near term. The first is in its fair use doctrine, which allows for certain use of in-copyright materials when their benefits to society outweigh the potential harm. Indeed, the HathiTrust collection's digitisation and maintenance has been successfully protected under the doctrine in Authors Guild, Inc. v. HathiTrust (S.D.N.Y.; Parker 2014) and Authors Guild v. Google, Inc. (S.D.N.Y.; Chin 2013). Notably, scholarly uses such as text analysis were noted as a transformative use in the Google case.

The second useful characteristic is a historical artefact of the United States' slow turn to copyright reform, resulting in copyright terms prior to 1978 that are determined by work publication date rather than author life. This means that copyright assessments are easier to do with the manifest publishing information contained within a book. There are a few aggregate-level generalisations that can be made. Prior to the Copyright Act of 1976, copyright was explicitly registered for 28 years, and renewable for a second term. We know that works published or registered before 1923 are almost certainly in the US public domain, because even with a renewal they would have lapsed into the public domain before copyright reform went into effect. Second, works published prior to reform in the 70 s have terms of 95 years, so we know that works published prior to 1926 have entered the public domain (by 2021), with that window moving each year. Finally, it is safe to assume that post-1963 works are in-copyright, given that the mechanisms for those to have entered the public domain are sparse. Only the period from 1926 to 1963 is more complicated, as the copyright status is dependent on whether a work's copyright registration was renewed.

Serving an international audience makes copyright determinations much more difficult, not only because of the complexity of honouring a myriad of regional differences, but also because many countries have copyright terms based on the author's death. Indeed, that is the case in the United States for works created since 1978, but countries that ratified the Berne Convention of 1886 have had that form of copyright term for a longer time (the United States joined the agreement over a century later). The issue for copyright determinations is that nearly every single work requires a secondary source of information, not contained within the book's content or metadata, to determine the author's life. At a scale of millions of works, and with many lesser-known works with obscure authors, there is no simple rule like the "pre-1926" rule in an author-based system. 
The HathiTrust addresses this issue by using a conservative worldwide public domain cutoff, maintaining that works published prior to 1881 are reasonably assumed to be in the public domain throughout the world. After that cutoff, while works may be public domain in various jurisdictions - for example, in Canada works by authors that died as recently as 1970 are in the public domain or 1950 for most European countries - making such determinations is a time-consuming process.

With over a century of works that the HathiTrust cannot assume to be globally available to the public, there is more of a burden to provide non-consumptive ways to benefit from the collection.

\section{Non-consumptive access}

Non-consumptive or non-expressive, access and use refers to use of materials in such a way that their use does not conflict with the rights of the material's copyright holders. In essence, the principle seeks to learn from text and disseminate those findings without making the original text available. In the case of non-consumptive access at the HathiTrust, the principle is further considered not only in the context of avoiding widespread dissemination of copyrighted work, but also in limiting the exposure between the library and the scholar.

Jockers, Sag and Schultz popularised the framing non-expressive use in an amicus brief filed on behalf of digital humanities scholars, in support of Google Books in Authors Guild v. Google, Inc. (2012). In that American litigation, the Authors Guild was challenging the legality of Google's right to digitise and maintain access to the scans of the Google Books project, similar to Authors Guild v. HathiTrust. Since the United States has a fair use doctrine, not explicitly laying out exceptions to copyright but defining criteria for judging when the benefits to society for use of copyrighted material outweigh the harm to the copyright holder, the amicus brief laid out the benefits to digital humanities.

The argument laid out by Jockers, Sag and Schultz was that non-expressive use is important to the "progress of science" in the digital humanities, that non-expressive aspects of works are not protected by copyright and finally, that the types of contributions that non-expressive text mining makes are precisely the types of advancements that fair use seeks to protect. The judge's opinion in that case made reference to this argument in a ruling in Google's favour, noting that "Google Books permits humanities scholars to analyze massive amounts of data - the literary record created by a collection of tens of millions of books. Researchers can examine word frequencies, syntactic patterns, and thematic markers to consider how literary style has changed over time" (The Authors Guild v. Google, Inc., S.D.N.Y.; Chin 2013).

Europe has no fair use doctrine protecting novel and productive uses of copyrighted material, making non-expressive use more critical (Hugenholtz 2013). 
A 2019 European Union directive finally clarified copyright exceptions related to text and data mining, though with limitations. Article 3 of the Directive on Copyright in the Digital Single Market (DSM) protects the rights of research institutions and cultural heritage institutions to preserve and reproduce copyrighted material, as well as permitting their use for text and data mining, while article 4 allows for the same outside of those institutions, albeit with the ability for copyright holders to opt out (European Parliament 2019).

At the HathiTrust, non-consumptive access is the term of art, as it makes clear the role of the scholar in working with materials. With a mediated collection, there are two practical parties in the discussion of copyright: one is the digital library, and their right to maintain works for research purposes, and the other is the external scholars that may seek to study that library's collection. The legal precedents in the United States and directives in the EU have focused on the first-party right to use material for text and data mining. The HathiTrust considers the relationship with third-party or affiliated second-party scholars, using non-consumptive access to refer to the form of that access. Per the HathiTrust's "Non-Consumptive Use Research Policy" (2017):

Non-consumptive Research (also called "non-consumptive analytics") means research in which computational analysis is performed on one or more volumes (textual or image objects) in the HT collection, but not research in which a researcher reads or displays substantial portions of an in-copyright or rights-restricted volume to understand the expressive content presented within that volume. Non-consumptive analytics includes such computational tasks as text extraction, textual analysis and information extraction, linguistic analysis, automated translation, image analysis, file manipulation, OCR correction, and indexing and search.

The HathiTrust outlines three of the modalities implemented by the HTRC: derived downloadable datasets, web-accessible data analysis and visualisation tools and HTRC Data Capsule. Datasets and analytic tools are described in detail over the next two sections.

\section{Extracted features}

The HTRC's EF Dataset is a dataset of all the books in the HathiTrust Digital Library, presented in a form that is useful for research but non-consumptive. The dataset shared a fingerprint of each page in the collection, such as which words occur and statistics on page layout such as line and character counts. Altogether, it shares information on 6.2 billion pages with 6.9 trillion words in a freely-available, openly-licensed dataset. To consider the value of this form of data, it is useful to consider its precedents. 
An area of research where access is challenged by copyright limitations is music information retrieval (MIR). MIR seeks to analyse and retrieve music by various sonic properties, for purposes such as music recommendation. Unlike the published word, there is little history for music that has lapsed into the public domain, given its relatively recent technological advent. Instead, the datasets of audio recordings collect from work where the artist has voluntarily dedicated their work to the public domain or, more commonly, given the work a permissive Creative Commons licence. The Free Music Archive, for example, has 106,000 tracks, comprising nearly one terabyte of date (Defferrard et al. 2017). Due to the limits on what can be distributed, however, larger music datasets are not distributed as audio recordings. Instead, datasets such as the Million Song Dataset (Bertin-Mahieux et al. 2011) share features of audio tracks.

A feature is an agnostic term for a countable property in machine learning. To analyse any sort of dataset, it needs to speak the quantified language of computing. Text and music are both examples of unstructured data, with no naturally countable structure. To a machine, a sentence is a string of characters, but there is no sense of what that sentence may communicate. In order to make sense of unstructured data, it first needs to be interpreted into one or a set of countable features. This process is called feature extraction. For music, extracted features may include properties such as the timing of all the beats in a song or the timbre, pitch and loudness of smaller segments of the song.

Feature extraction is a necessary step of working with unstructured data: researchers first need to derive a malleable representation of the document, then they may look to analytic processes such as classification, clustering, tagging, generation etc. The clever approach taken by the Million Song Dataset is in distributing already extracted features, with songs segmented and counted in useful ways (Bertin-Mahieux et al. 2011). This allows distribution of useful information about songs - in many cases the very information that a researcher would have extracted from an audio file - without actually distributing a listenable track that can be enjoyed in a way that may be seen as harmful to the original creator. While there is value in the mechanics of extraction - for example, a scholar may want a different algorithm for finding beats or splitting texts into words -this type of dataset benefits a large proportion of uses. Further, it enables access to data that might otherwise not be distributable.

Inspired by datasets from the field of MIR, the HTRC developed and released the HTRC EF Dataset (Organisciak et al. 2017, Jett et al. 2020). The EF Dataset distributes book-level and page-level features for each of the volumes in the HathiTrust Digital Library. The derived dataset approach has also been implemented for other massive-scale text digital libraries: Google's Ngrams Viewer offered datasets of term counts at the full collection level (Michel et al. 2011) and JSTOR's Data for Research service "includes metadata, n-grams, and word counts" for the majority of JSTOR's articles, chapters and reports. 
In text mining and analysis, one feature stands above all others: the token count. Commonly, a text is split up, or tokenised, into sentences and those sentences into individual words, or more generally tokens, which may be counted. Page-level token counts are the cornerstone feature in the EF Dataset. For every page of every book, the text is tokenised, the tokens are tagged by part of speech, and the count of each type of use for each word is provided. The part of speech tagging will differentiate between the verb rose (to rise) and the noun rose (flower). Further, counts are provided for their original casing, so the name Rose will be differentiated from the lower-cased flower, except when the flower is used at the start of a sentence; in that instance, the part-of-speech tagging will still differentiate between a proper noun and noun.

Token counts are a type of representation known as bag-of-words. The intuition is that words represent a topical fingerprint of what a text is about. This simplifying assumption fails in certain contexts, such as negation; for example, "not good" is a phrase that would lose its meaning when the words are considered independently of each other. Yet, when paired with language models that can represent similarity between words (e.g., that "dog" and "canine" discuss similar topics) and term weighting approaches that acknowledge that not all words are equally informative, they can effectively support many text classification, clustering and analysis workflows. For example, Underwood and Sellers used the HathiTrust data to identify the emergence of a style associated with prestigious literature, noting an increased tendency for work reviewed by prominent periodicals to exaggerate stylistic features from prestigious work before it (2016).

With respect to non-consumptive access, the lack of positional information is an asset, enabling access to works that would otherwise be too legally risky to distribute otherwise. Yet, the token count approach does not satisfy all scholarly needs, such as text modelling approaches that learn text based on a word's context in a larger sequence, like BERT (Devlin et al. 2018) or ELMO (Peters et al. 2018). Further, artificial neural network-based approaches are challenged by the use of "words" as their token unit. Counting every unique word can result in counts for hundreds of thousands or millions of words, a large vocabulary. Since neural network approaches generally allow for improved performance to be eked out from larger, more parameter-heavy models, the cost of supporting a large vocabulary may be seen as a less favourable way to allocate computing resources. Rather, words are often encoded using sub-word representations such as byte-pairencoding (Gage 1994, Sennrich et al. 2016), which has a smaller vocabulary of character sequences and a word may be represented through multiple tokens. In the context of feature datasets, the trade-off may be necessary, as the lack of positional information is a central contributor towards enabling non-consumptive access. Yet, given the direction of natural language processing research, which often precede trends in information science, information retrieval and eventually the digital humanities, a productive area 
of future research would be in exploring new forms of practical but nonconsumptive features for text.

Beyond token counts, the EF Dataset includes other valuable features, including:

- $\quad$ key metadata for each volume, including author, date, publisher and place, language, classification numbers, control numbers and volume enumeration information. Descriptive metadata is available from other HathiTrust sources, but is included with the EF Dataset for convenience;

- disambiguation of headers and footers from content. This allows researchers to easily ignore text that repeats on multiple pages, which may confound a text mining model;

- counts of sentences in the text as well as the number of lines from the top to bottom of a page. This can be useful information for determining the type of content on a page. For example, front matter - content like title pages and dedications, which researchers may be less interested in - tends to have fewer lines of text;

- counts of characters on the leftmost and rightmost side of a page. This feature speaks to the physical structure of a page and how the written word interacts with it, and may be useful for identifying what type of page is being dealt with. A title page will have statistically more capitalised characters on the left, a table of content will have the same as well as roman or Arabic numerals on the right. Poetry is laid out with more regard for layout than prose so some types of poetry may be identifiable through this feature;

- algorithmically inferred language per page. Sometimes cataloguing metadata is incorrect or incomplete, other times books contain multiple languages and a finer approach for language is needed; this feature provides a second guess of each page's language.

The limitations of a feature dataset are that it takes the flexibility of parameterising and choosing feature representations away from the scholar. It may also be, as Samberg and Hennesy note, "puzzling" for scholars hoping to work with a txt or PDF file (2019), or those that work with fully automated tools that internally expect to do feature extraction and analysis all at once. Yet, its ability to open the door to scholarship over sensitive data should not be overlooked, and it is seeing adoption in other digital library spheres.

\section{HathiTrust+Bookworm}

While the EF Dataset frees a scholar from part of a typical text analysis workflow, it's use is still burdened by economies of scale and exploration and inference require technical expertise, time and computational resources. At the same time, for a scholar that is new to text analysis the 
abstracted nature of the EF Dataset may be off-putting, as not being able to review the original texts may lend a sense of lost sensitivity to the underlying materials.

With HT+BW, the HTRC seeks to promote non-consumptive use of the collection for a different set of uses and users. HT+BW is built as a tool for exploratory data analysis (Tukey 1977) over the HathiTrust collection, allowing quick, flexible and robust queries to be made of the corpus and visualized.

Underlying $\mathrm{HT}+\mathrm{BW}$ is Bookworm, a tool for visualising language trends and corpus trends at large scale. It is best to understand Bookworm as a quantitative API (application programming interface), a tool that allows you to form complex queries and receive a structured data response. Paired with the scale and the professionally catalogued metadata of the Hathitrust, Bookworm becomes a powerful way of studying history, language and culture in the published word.

Bookworm queries are comprised of a few components:

- Faceting: Faceting is the disambiguation of results by a specific property of result. Consider a question of "how many books are in the collection?" This query would have one number as a result. Now, consider a facet such as date. For a question such as "how many books are in the collection, by year", our results would have a count for every single year seen in the collection. This type of facet can be applied for many properties, such as Library of Congress Classification System class or subclass, publication country, publication state (for USA, United Kingdom or the former USSR), author, copyright status and so on. This can be very powerful, especially when multiple facet groups are used.

- Examples: "how many books are in the collection, by subject class and by publication year?"; "how often is the word 'gender' used in different publication countries?"

- Filtering: Filtering similarly uses book or document metadata, though for selecting texts of interest, rather than for disaggregating results. You can still facet on the results, for example, seeing the prevalence of "data" by year, but unlike Bookworm's precedent Google NGrams Viewer, you are not limited to searching over the entire corpus.

- Examples: "How often is the word 'data' used in the Library Sciences?"; "How has 'creativity' emerged in the 20th Century?"

- Word Searching: A scholar can specify a word to look for in the full or filtered collection. In essence, this is a type of filter, applied on the content of the underlying corpus rather than the metadata. While word search is optional - without it a scholar can still observe bibliographic metadata trends - this is the functionality that truly enables scholars to glimpse inside books in a non-consumptive manner.

- Example: "When did the word 'Inuit' replace the non-preferred 'Eskimo' in Canadian books?" 
- Output Statistics: A query can ask for results in various ways. These include "words per million", a ratio of how much a specified word occurs out of every million words; "text percent", a percentage of books in the facet that have a specified word; and "text count", which is the nominal number of books in each facet of the query, including a word if it was specified.

- Examples: "How many books discuss 'freedom'?", "Proportionally, how often in the language is 'freedom' used?"

With these components, HT+BW is able to answer complex queries, returning raw data results or visualizing them. The "main" view of HT+BW, the Bookworm GUI (http://bookworm.htrc.illinois.edu), visualises word trends with a time-series line chart. In this view, the faceting group is "date", so that all results are disaggregated by year, and the word filter is specified by the user, as with a related preceding tool, Google Ngrams Viewer. Where the Bookworm GUI view differentiates itself is in allowing more detailed filtering. Rather than only asking a question of the form, "how has the usage of X changed over time", it is possible to ask questions such as "how has the usage of X changed in a specific country/subject class/literary form/etc.". This also allows the same term to be compared in two settings, such as "how has the usage of 'cookie' changed in general texts vs. technology texts".

While the Bookworm GUI includes faceting by date, it is not the only way to query the collection, and the HTRC offers other tools to visualize the responses to those types of questions. For example, it may be necessary to facet by two groups - perhaps date and class. For such queries, the Bookworm Advanced interface (https://bookworm.htrc.illinois. edu/advanced) offers modules for graphics such as heatmaps - a twodimensional grid where each $x-y$ grid square is coloured - or stacked line charts. Bookworm Advanced also offers scatter plots, maps, and bar charts, using a declarative style where a scholar can bind data elements to visual elements (Wilkinson 1999), specifying which data is bound to the $x$-axis, $y$-axis, colour, fill or size of an element. For a template-based alternative, maps and heatmaps may also be customised in the Bookworm Playground (https://bookworm.htrc.illinois.edu/app).

These interfaces provide different ways to work with the same underlying API. Their intent is to balance expressiveness, conciseness and accessibility for visualising the data. To make the system work for complex cases as well as playful, exploratory ones, the interfaces offered by HT+BW vary in how many decisions have been made in the interface and how many decisions are left to the user. The non-consumptive "masking" of sensitive data is designed into the core API, so these interfaces do not require special considerations in their implementation. This approach also allows for the API to be the most advanced option for scholarship. The HT+BW API is entirely open, and researchers can craft their own custom queries through a web browser or by using a statistical programming library like Python or R. 
$\mathrm{HT}+\mathrm{BW}$ is a specific implementation of an open-access tool, Bookworm, against the HathiTrust collection. Bookworm was founded at the Harvard Cultural Observatory by Erez Lieberman Aiden and Jean-Baptiste Michel, following from their earlier work on the Google Ngrams Viewer (Aiden and Michel 2014). The Google Ngrams Viewer does not offer the same flexibility and metadata richness as $\mathrm{HT}+\mathrm{BW}$, primarily allowing a single view - time series line chart - over the entire collection. However, it $i$ non-consumptive, an early and enlightening example of how massive datasets can be shown to people, and not only described. It is still being maintained, with an update in 2020, and serves as a good additional case of this form of exploratory data analysis tool. It has one primary strength over HT+BW: the inclusion of longer phrases (n-grams), whereas HT+BW is focused on unigrams. Its notable approachability has been influential, such as with FiveThirtyEight's tool for exploring a data dump of the reddit online community, "How the Internet Talks" (King and Olson 2015). Bookworm development has been led by Benjamin M. Schmidt (2011) for the past few years, and HT+BW's large-scale improvements were funded by the National Endowment for the Humanities (\#HK-50176-14) and led by Schmidt and this chapter's authors.

$\mathrm{HT}+\mathrm{BW}$ is a demonstrative example of Bookworm's functionality, but not the only one. Other institutions can freely make use of Bookworm, including the large-scale improvements developing for supporting a collection at the scale of the HathiTrust collection. This may be especially appropriate for institutions working to make their own collections of sensitive data accessible, whether they are copyrighted texts or simply texts that for various reasons a group may not want to distribute fully.

\section{Other approaches}

As we see with the multi-pronged approach to interfacing with $\mathrm{HT}+\mathrm{BW}$, so goes the broader HTRC strategy. In resolving to provide non-consumptive access and eluding direct access to raw text, the Center faces a challenge: that the scholars they serve have varied methodological approaches and research questions, and no single approach outside can meet that litany of needs. Practically, solutions such as a feature dataset or exploratory data analysis tool may meet enough needs to allow institutions or researchers to mediate access to sensitive materials. However, given its broad mission, the HTRC implements multiple strategies in order to minimise the use cases that cannot be met. As such, it is an interesting case study, offering different views of how non-consumptive research may be performed and the strengths and weaknesses of each.

Beyond the EF Dataset and HT+BW, the HTRC's other non-consumptive offerings are the Portal, a web interface for text mining tools; the Data Capsule, a secure virtual machine for research and Advanced Collaborative Support (ACS), a program for partnering Center staff with scholars. 
The HTRC Portal is a web-based toolkit that implements analytic tools in-browser. A scholar may select a custom subset of the HathiTrust Digital Library and apply an out-of-the-box algorithm to it in a custom job, such as named entity recognition and token counting. A user of the Portal specifies what they want and how, then receives the processed output.

A Portal approach has some advantages. It is easy to use and, in existing solely on the web, does not require much effort to learn. However, it is challenging to maintain and heavily restricts the types of uses on the data - each classifier, tokeniser, analyser needs to be explicitly prepared. To address this challenge, HTRC early on implemented a module-based platform, Software Environment for the Advancement of Scholarly Research (SEASR). SEASR uses a piped workflow paradigm (Ács et al. 2010), where a developer creates an algorithm by connecting modules, from input through to preprocessing, feature extraction, analysis and output. Still, user demand for more flexibility combined with the person-power required to manage the system has led to a partial deemphasizing of the Portal, which functions most prominently as an educational tool and an entryway to other non-consumptive tools.

Providing greater flexibility is the Data Capsule, which provides a secure, sanitised virtual machine for working with original, sensitive texts. The Data Capsule runs a Linux desktop environment on HTRC servers, into which an approved scholar connects. Once there, the system runs in two modes: maintenance mode and secure mode. In maintenance mode, the server is connected to the web and can install software or download datasets from the internet. However, no HathiTrust data is accessible, beyond a small sample set. When a scholar flips to secure mode, web connectivity in the virtual machine is blocked, so nothing can be brought in or taken out, but the HathiTrust Digital Library book data is mounted and accessible. The intent is for maintenance mode to be where research code and workflows are developed and secure mode where they are run.

The Data Capsule requires a stronger partnership with researchers as well as more supervision of their work. Only researchers from institutions with a legal partnership with the HathiTrust may process in-copyright texts, and all results that are intended to be removed from the data capsule must undergo a manual human review, to ensure that only non-expressive outputs are taken out. In essence, the Data Capsule is a digital version of a restricted research study or archive, where materials can only be handled on "location". The Data Capsule has some obstacles to adoption by other institutions, including time, manpower and computational costs. It needs to be hosted somewhere, is a complex paradigm engineering-wise, and requires staffing for moderation of exported results. In exchange for those hurdles, it offers the most flexibility for advanced scholarship, such as work on understanding gendered language in literature (Underwood, Bamman, and Lee 2018), and the study of books through the lens of critical reviews (Underwood 2020). 
Each non-consumptive strategy taken by the HTRC balances a tradeoff between access and flexibility. If these approaches are not sufficient, the HTRC offers a more bespoke approach through its ACS program. ACS is a series of grants of HTRC staff time. Scholars apply with projects that may not be tractable using publicly available tools and datasets and, if awarded, the HTRC will work with them on the research, including being the intermediary between the researcher and any in-copyright data. The program has supported a number of digital humanities and information science projects, such as a study of the influence of the Chicago school of architecture (Baciu 2019) and work into automated segmentation of book structure (McConnaughey, Dai, and Bamman 2017).

\section{Broader impact}

Non-consumptive or non-expressive use is a principle of scholarly research that considers ways for a work to be studied without revealing the original, copyrighted text. It can refer both to the product of research and primary source for research. In some jurisdictions, text mining applications that disseminate non-expressive results are explicitly or implicitly exempted from copyright law. However, this is complicated when multiple parties are involved. Whereas a scholar can perform text analysis on personally-obtained raw text, they cannot disseminate the original texts for reproducibility, nor can digital libraries share it in the name of access for scholars. For institutions and scholars seeking to improve access to privileged or sensitive materials, non-consumptive by design offers a path forward.

The HTRC has a mission to support research access to the extremely rich HathiTrust Digital Library collection, but that collection is two-thirds in-copyright. By the nature of that conundrum, the HTRC's services have had to be non-consumptive by design. Digital libraries and other research collections continue to grow, while text and data mining mature as methods in the digital humanities, computational social sciences and information science. If that growth continues - as the increasing investment in data as a service by academic publishers would suggest - it will be important for scholarly infrastructures to adapt to the increased value and demand for aggregate-level access to archival collections. The HTRC provides a template on how collections with privileged materials can balance ease of access with legal, ethical or proprietary restrictions.

\section{Bibliography}

Ács, Bernie, Xavier Llorà, Loretta Auvil, Boris Capitanu, David Tcheng, Mike Haberman, Limin Dong, Tim Wentling, and Michael Welge. 2010. "A General Approach to Data-Intensive Computing Using the Meandre Component-Based 
Framework." In Proceedings of the 1st International Workshop on Workflow Approaches to New Data-Centric Science (WANDS 10), 1-12. New York, NY: Association for Computing Machinery. https://doi.org/10/dv3j3s

Aiden, Erez and Jean-Baptiste Michel. 2014. Uncharted: Big Data as a Lens on Human Culture. New York, NY: Riverhead Books.

Baciu, Dan C. 2019. "Chicago Schools: Large-Scale Dissemination and Reception." Prometheus 2 (2). 20-43.

Bamman, D., M. Carney, J. Gillick, C. Hennesy, and V. Sridhar. 2017. "Estimating the Date of First Publication in a Large-Scale Digital Library." In ACM/IEEE Joint Conference on Digital Libraries (JCDL), 1-10. https://doi.org/10.1109/ JCDL.2017.7991569

Bertin-Mahieux, Thierry, Daniel PW Ellis, Brian Whitman, and Paul Lamere. 2011. "The Million Song Dataset." In Proceedings of the 12th International Society for Music Information Retrieval Conference, 2:10. Miami, FL.

Burrows, J. F. 1987. "Word-Patterns and Story-Shapes: The Statistical Analysis of Narrative Style." Literary and Linguistic Computing 2 (2): 61-70. https://doi. org/10.1093/11c/2.2.61

Burton, D. M. 1981. "Automated Concordances and Word Indexes: The Fifties." Computers and the Humanities 15 (1): 1-14. https://doi.org/10/btssqs.

Chin, Denny. 2013. The Authors Guild v. Google, Inc. S.D.N.Y.

Common Crawl. 2021. "Common Crawl." https://commoncrawl.org/

Darnton, Robert. 2013. "The National Digital Public Library Is Launched." The New York Review of Books 25. https://nybooks.com/articles/2013/04/25/ national-digital-public-library-launched

Defferrard, Michaël, Kirell Benzi, Pierre Vandergheynst, and Xavier Bresson. 2017. "FMA: A Dataset for Music Analysis." In International Society for Music Information Retrieval Conference (ISMIR).

Devlin, Jacob, Ming-Wei Chang, Kenton Lee, and Kristina Toutanova. 2018. "BERT: Pre-Training of Deep Bidirectional Transformers for Language Understanding," October. https://arxiv.org/abs/1810.04805v2.Archiv.org

European Parliament. 2019. "Directive (EU) 2019/790 of the European Parliament and of the Council of 17 April 2019 on Copyright and Related Rights in the Digital Single Market and Amending Directives 96/9/EC and 2001/29/EC." Official Journal of the European Union, May. https:/eur-lex.europa.eu/eli/dir/2019/790/oj

Freeland, Chris. 2021. "Internet Archive's Modern Book Collection Now Tops 2 Million Volumes." Internet Archive Blogs (blog). February 3, 2021. https:// blog.archive.org/2021/02/03/internet-archives-modern-book-collection-nowtops-2-million-volumes/

Gage, Philip. 1994. "A New Algorithm for Data Compression.” C Users Journal 12 (2): $23-38$.

Geiger, Christophe, Giancarlo Frosio, and Oleksandr Bulayenko. 2018. "The Exception for Text and Data Mining (TDM) in the Proposed Directive on Copyright in the Digital Single Market - Legal Aspects.” SSRN Scholarly Paper ID 3160586. Rochester, NY: Social Science Research Network. https://doi. org/10.2139/ssrn.3160586

HathiTrust. 2017. "Non-Consumptive Use Research Policy." HathiTrust Digital Library. https://www.hathitrust.org/htrc_ncup

HathiTrust. 2021a. "About." HathiTrust Digital Library. https://www.hathitrust. org/about 
HathiTrust. 2021b. "HathiTrust Dates." HathiTrust Digital Library. https://www. hathitrust.org/visualizations_dates

HathiTrust. 2021c. "HathiTrust Languages.” HathiTrust Digital Library. https:// www.hathitrust.org/visualizations_languages

Hockey, Susan. 2004. "The History of Humanities Computing." In Companion to Digital Humanities, edited by Susan Schreibman, Ray Siemens, and John Unsworth. Oxford: Blackwell Publishing Professional. http://www.digitalhumanities.org/ companion/

Holmes, D. I. 1985. "The Analysis of Literary Style - A Review." Journal of the Royal Statistical Society: Series A (General) 148 (4): 328-41. https://doi.org/10/ bghv3t

Hugenholtz, P. Bernt. 2013. "Fair Use in Europe." Communications of the ACM 56 (5): 26-28. https://doi.org/10/gjfvd3

Jessen, Jenica. 2020. "Looking Back on 2020." Internet Archive Blogs (blog). December 19, 2020. https://blog.archive.org/2020/12/19/looking-back-on-2020/

Jett, Jacob, Boris Capitanu, Deren Kudeki, Timothy W. Cole, Yuerong Hu, Peter Organisciak, Ted Underwood, Eleanor Dickson Koehl, Ryan Dubnicek, and J. Stephen Downie. 2020. "The HathiTrust Research Center Extracted Features Dataset (2.0).” https://doi.org/10.13012/R2TE-C227

Jockers, Matthew, Matthew Sag, and Jason Schultz. 2012. "Brief of Digital Humanities and Law Scholars as Amici Curiae in Authors Guild v. Google." SSRN Scholarly Paper ID 2102542. Rochester, NY: Social Science Research Network. https://doi.org/10.2139/ssrn.2102542

JSTOR. n.d. "JSTOR Data for Research.” JSTOR. Accessed February 5, 2020. https://www.jstor.org/dfr/

King, Ritchie, and Randy Olson. 2015. "How the Internet* Talks.” FiveThirtyEight. Last updated September. 22, 2017. https://projects.fivethirtyeight.com/redditngram/

Lazer, David, Alex (Sandy) Pentland, Lada Adamic, Sinan Aral, Albert Laszlo Barabasi, Devon Brewer, Nicholas Christakis, et al. 2009. "Life in the Network: The Coming Age of Computational Social Science.” Science 323 (5915): 721-23. https://doi.org/10/c9w2g3

LexisNexis. 2003. “The LexisNexis Timeline.” http://www.lexisnexis.com/anniversary/30th_timeline_fulltxt.pdf

Manovich, Lev. 2009. "Cultural Analytics: Visualising Cultural Patterns in the Era of 'More Media." Domus (March 2009). Milan.

McConnaughey, Lara, Jennifer Dai, and David Bamman. 2017. "The Labeled Segmentation of Printed Books." In Proceedings of the 2017 Conference on Empirical Methods in Natural Language Processing, 737-47. Copenhagen, Denmark: Association for Computational Linguistics. https://doi.org/10/ggc2kq

Michel, Jean-Baptiste, Yuan Kui Shen, Aviva Presser Aiden, Adrian Veres, Matthew K. Gray, Joseph P. Pickett, Dale Hoiberg, et al. 2011. "Quantitative Analysis of Culture Using Millions of Digitized Books.” Science 331 (6014): 176-82. https:// doi.org/10.1126/science.1199644

Milligan, Ian. 2016. "Lost in the Infinite Archive: The Promise and Pitfalls of Web Archives." International Journal of Humanities and Arts Computing 10 (1): 78-94. https://doi.org/10/gjgnw7

Milligan, Ian. 2019. History in the Age of Abundance? How the Web Is Transforming Historical Research. Montreal, QC: McGill-Queen's University Press. 
Moretti, Franco. 2013. Distant Reading. London: Verso Books.

Nelson, Laura K. 2014. "The Power of Place: Structure, Culture, and Continuities in U.S. Women's Movements.” PhD diss. UC Berkeley. https://escholarship.org/uc/ item/8794361r

Organisciak, Peter, Boris Capitanu, Ted Underwood, and J. Stephen Downie. 2017. "Access to Billions of Pages for Large-Scale Text Analysis." In iConference 2017 Proceedings Vol. 2. Wuhan, China: iSchools. https://doi.org/10.9776/17014

Organisciak, Peter, Summer Shetenhelm, Danielle Francisco Albuquerque Vasques, and Krystyna Matusiak. 2019. "Characterizing Same Work Relationships in Large-Scale Digital Libraries." In International Conference on Information, 419-25. Cham, Switzerland: Springer.

Osterberg, Gayle. 2017. "Update on the Twitter Archive at the Library of Congress. Library of Congress Blog." Last updated December 26, 2017.//blogs.loc.gov/ loc/2017/12/update-on-the-twitter-archive-at-the-library-of-congress-2/

Palmer, Carole L., Oksana L. Zavalina, and Megan Mustafoff. 2007. "Trends in Metadata Practices: A Longitudinal Study of Collection Federation." In Proceedings of the 7th ACM/IEEE-CS Joint Conference on Digital Libraries, 386-95. JCDL '07. New York, NY: Association for Computing Machinery. https:// doi.org/10/djcbw8

Parker, Barrington Daniels Jr. 2014. Authors Guild, Inc. v. Hathitrust. S.D.N.Y.

Pechenick, Eitan Adam, Christopher M. Danforth, and Peter Sheridan Dodds. 2015. "Characterizing the Google Books Corpus: Strong Limits to Inferences of Socio-Cultural and Linguistic Evolution.” PLoS One 10 (10): e0137041. https:// doi.org/10.1371/journal.pone. 0137041

Peter Suber. 2005. "The Open Content Alliance." SPARC Open Access Newsletter, November 2, 2005. https://dash.harvard.edu/bitstream/handle/1/4552008/suber_ oca.htm?sequence $=1$

Peters, Matthew E., Mark Neumann, Mohit Iyyer, Matt Gardner, Christopher Clark, Kenton Lee, and Luke Zettlemoyer. 2018. "Deep Contextualized Word Representations.” ArXiv:1802.05365 [Cs], February. http://arxiv.org/abs/1802.05365

Purday, Jon. 2009. "Think Culture: Europeana.eu from Concept to Construction." The Electronic Library 27 (6): 919-37. https://doi.org/10/c4mkkp

Ross, Stephen, and Jentery Sayers. 2014. "Modernism Meets Digital Humanities." Literature Compass 11 (9): 625-33. https://doi.org/10/gmxdw9

Samberg, Rachael Gayza, and CodyHennesy. 2019. "Law and Literacy in NonConsumptive Text Mining: Guiding Researchers Through the Landscape of Computational Text Analysis." In Copyright Conversations: Rights Literacy in a Digital World, Sara R. Benson, ed. Chicago, IL: Association of College and Research Libraries. https://escholarship.org/uc/item/55j0h74g

Schmidt, Benjamin M. 2011. "Bookworm.” Beta Sprint Competition Selection presented at the Digital Public Library of America Plenary Meeting, Washington, DC, October 21: Digital Public Library of America.

Schofield, Alexandra, Laure Thompson, and David Mimno. 2017. "Quantifying the Effects of Text Duplication on Semantic Models." In Conference on Empirical Methods on Natural Language Processing. Copenhagen, Denmark. http://www. cs.cornell.edu/ xanda/textduplication2017.pdf

Sennrich, Rico, Barry Haddow, and Alexandra Birch. 2016. "Neural Machine Translation of Rare Words with Subword Units." ArXiv:1508.07909 [Cs], June. http://arxiv.org/abs/1508.07909 
Sprokel, Nico. 1978. "The 'Index Thomisticus.” Gregorianum 59 (4): 739-50.

Tukey, John W. 1977. Exploratory Data Analysis. Reading, MA: Addison-Wesley Publishing Company. http://www.ru.ac.bd/wp-content/uploads/sites/25/2019/ 03/102_05_01_Tukey-Exploratory-Data-Analysis-1977.pdf

Underwood, Ted. 2017. "A Genealogy of Distant Reading." Digital Humanities Quarterly 011 (2). http://www.digitalhumanities.org/dhq/vol/11/2/000317/000317. html

Underwood, Ted, and Jordan Sellers. 2016. "The Longue Durée of Literary Prestige." Modern Language Quarterly 77 (3): 321-44. https://doi.org/10.1215/ 00267929-3570634

Underwood, Ted. 2020. "There's No Such Thing as Bad Publicity: Toward a Distant Reading of Reception." Paper Presented at MLA 2020. Seattle, USA: Modern Language Association.

Underwood, Ted, David Bamman, and Sabrina Lee. 2018. "The Transformation of Gender in English-Language Fiction." Journal of Cultural Analytics 1 (1) (February 13, 2018): 11035. https://doi.org/10/gjjz7h

Unsworth, John. 2004. "Forms of Attention: Digital Humanities Beyond Representation" In Third Conference of the Canadian Symposium on Text Analysis (CaSTA), Hamilton, ON: McMaster University. https://johnunsworth. name/FOA/

Wilkinson, Leland. 1999. The Grammar of Graphics. New York, NY: Springer-Verlag. 